\title{
Novel Dual-Band Design of Planar Dipole Array for 4G LTE/WiMAX Access Points
}

\author{
Jui-Han Lu and Shi-Wei You \\ Department of Electronic Communication Engineering, National Kaohsiung Marine University, Kaohsiung 811, Taiwan \\ Correspondence should be addressed to Jui-Han Lu, jhlu@webmail.nkmu.edu.tw
}

Received 22 July 2011; Accepted 23 August 2011

Academic Editor: Dau-Chyrh Chang

Copyright ( $) 2011$ J.-H. Lu and S.-W. You. This is an open access article distributed under the Creative Commons Attribution License, which permits unrestricted use, distribution, and reproduction in any medium, provided the original work is properly cited.

A novel planar $1 \times 4$ dipole array with dual-band operation for LTE/WiMAX access points is proposed. The impedance bandwidths, determined from VSWR $\leqq 2.0$, can reach about 525/747 MHz $(20.2 / 21.3 \%)$ for the $2.6 / 3.5 \mathrm{GHz}$ operating bands, respectively, which are covering the required bandwidth for LTE $(2.5 \sim 2.7 \mathrm{GHz})$ and WiMAX $(3.3 \sim 3.7 \mathrm{GHz})$ system. This proposed dualband dipole array also provides maximum peak antenna gains and efficiencies of 7.3/7.5 dBi and 89/75\% across $2.6 / 3.5 \mathrm{GHz}$ bands, respectively, with good omnidirectional radiation pattern in XY-plane.

\section{Introduction}

Recently, LTE (Long-Term Evolution) and WiMAX (Worldwide Interoperability for Microwave Access) systems for the Fourth-Generation (4G) mobile communication has attracted high attention for broadband access in wireless metropolitan area network (WMAN) environment. For the LTE/ WiMAX base stations or access points, the high-gain array antenna with omnidirectional radiation in the azimuthal plane is usually required for long-distance communication. The related array designs had been presented by using two $1 \times 2$ dipole arrays [1], collinear array antenna with three in-phase half-wavelength radiating elements [2], and fourelement collinear array antenna [3]. However, these above array antennas were focused on the single-band operation. For dual-band operation, only the array design [4] had been proposed by using one dipole antenna for $2.4 \mathrm{GHz}$ and $1 \times 2$ array antenna for $5.2 \mathrm{GHz}$ WLAN communication. And, there was a disadvantage of lower antenna gain (less than $4 \mathrm{dBi}$ ), which was unsuitably in the practical applications for LTE/WiMAX access points. Meanwhile, dual-band dipole array for LTE/WiMAX applications is very scant in the open literature. Therefore, in this paper, we propose a novel planar dual-band dipole array mainly comprises two $1 \times$ 4 dipole arrays arranged back to back, and can be easily constructed by printing on both sides of a dielectric substrate (see the geometry shown in Figure 1). By properly adjusting the arms' lengths of each unsymmetrical dipole element, the operating bandwidths (VSWR $\leqq 2.0$ ) can reach about $525 / 747 \mathrm{MHz}(20.2 / 21.3 \%)$, which are enough for LTE and WiMAX system. Also, the proposed dipole array provides maximum peak antenna gains and efficiencies of 7.3/7.5 dBi and $89 / 75 \%$ across $2.6 / 3.5 \mathrm{GHz}$ bands, respectively, with good omnidirectional radiation pattern in the azimuthal plane and narrow-beamwidth pattern in the elevation plane. Details of the proposed dual-band dipole array designs are described, and experimental results for the obtained highgain performance are presented and discussed.

\section{Antenna Design and Experimental Results}

Figure 1 illustrates the geometry of the proposed omnidirectional dipole array antenna for 2.6/3.5 GHz LTE/WiMAX access points. The dimensions of the feeding network composed of the in-phase power divider with multisection binomial quarter wave transformers are shown in Figure 2. The proposed dual-band array is fed by a $50 \Omega$ coaxial cable line at the center of the $100 \Omega$ microstrip line (point O) which is etched on the both printed sides of an inexpensive FR4 substrate with the volume of $280 \times 31 \times 0.8 \mathrm{~mm}^{3}$, dielectric constant $\varepsilon_{r}=4.7$ and loss tangent $\tan \delta=0.0245$. 


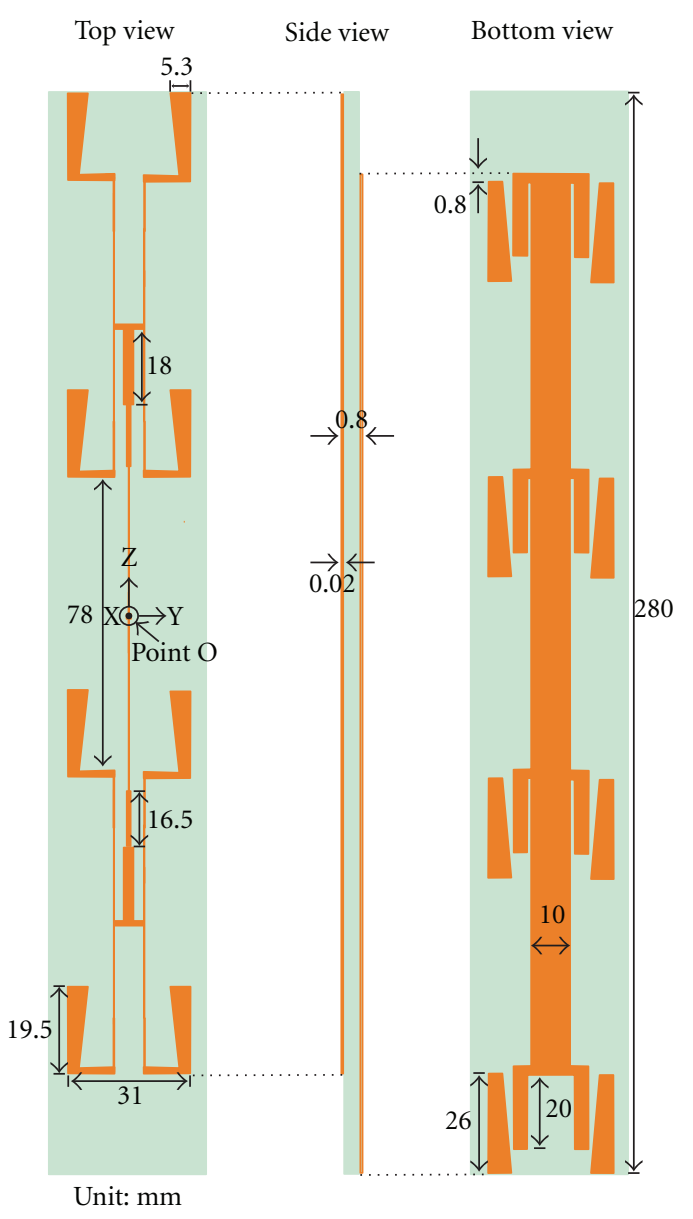

FIGURE 1: Geometry of the proposed planar dual-band dipole array with high-gain operation.

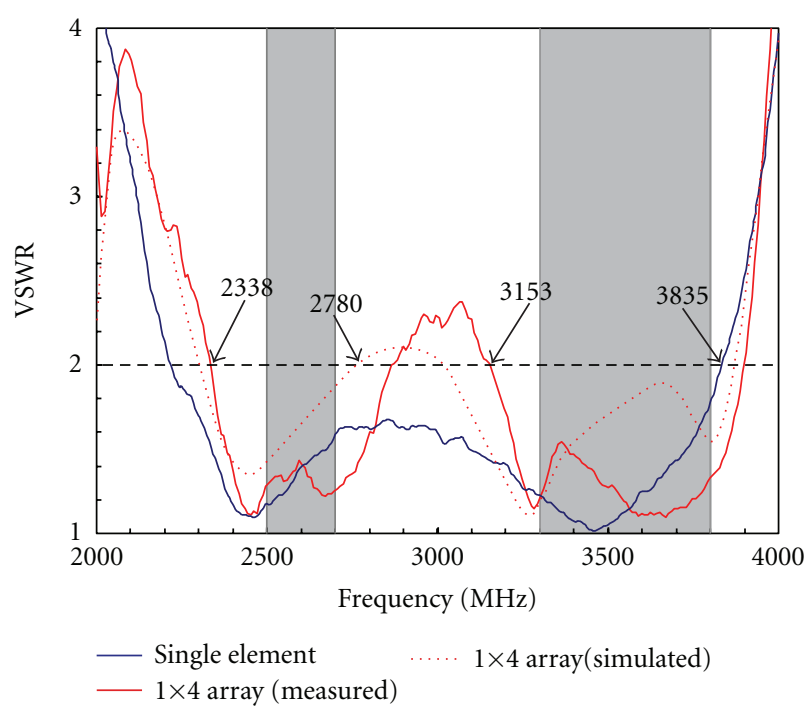

FIGURE 2: Simulated and measured VSWR against frequency for the proposed planar dual-band dipole array.
TABLE 1: Simulated and measured VSWR against frequency for the proposed planar dual-band dipole array with high-gain operation.

\begin{tabular}{lcccc}
\hline Proposed array & $\begin{array}{c}f_{1 L} \sim f_{1 H} \\
(\mathrm{MHz})\end{array}$ & $\begin{array}{c}\text { B.W. } \\
(\mathrm{MHz} / \%)\end{array}$ & $\begin{array}{c}f_{1 L} \sim f_{1 H} \\
(\mathrm{MHz})\end{array}$ & $\begin{array}{c}\text { B. W. } \\
(\mathrm{MHz} / \%)\end{array}$ \\
\hline \multirow{2}{*}{ Simulated } & $2298 \sim$ & \multirow{4}{*}{$482 / 18.5$} & $3013 \sim$ & 3872 \\
& 2780 & & $859 / 24.5$ \\
Measured & $2338 \sim$ & $525 / 20.2$ & $\begin{array}{c}3153 \sim \\
3900\end{array}$ & $747 / 21.3$ \\
\hline
\end{tabular}

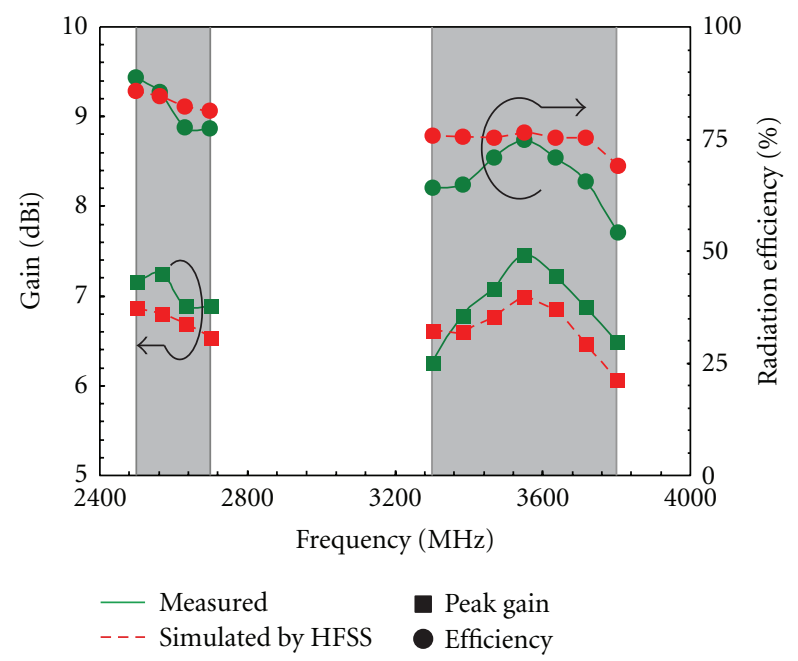

FIGURE 3: Simulated and measured peak gains and efficiencies across the operating bands for the proposed dual-band dipole array.

This proposed planar dual-band array is comprised of four dipole back-to-back elements with the spacing of $78 \mathrm{~mm}$, which are arranged symmetrically with respect to the central narrow rectangular ground plane of width $10 \mathrm{~mm}$, printed on the rear surface of the FR4 substrate to obtain omnidirectional radiation pattern in the azimuthal plane. In this study, by introducing the inverted U-shaped strip with the length of $20 \mathrm{~mm}$ in the ground plane to form the unsymmetrical dipole element, the resonant mode operating at $3.5 \mathrm{GHz}$ band can be excited due to the resonant length of the unsymmetrical dipole chosen to be corresponding approximately to 0.46 operating wavelength of $3.5 \mathrm{GHz}$ band. And, a parasitic strip with the length of $26 \mathrm{~mm}$ is arranged parallel with the inverted $U$-shaped ground strip as the lower arm of the printed dipole element for the excitation of $2.6 \mathrm{GHz}$ operating band with the resonant length of $45.5 \mathrm{~mm}$, which is less than that of the conventional halfwavelength dipole to have compact operation.

To demonstrate the above deduction and guarantee the correctness of simulated results, the electromagnetic simulator HFSS based on the finite element method [5] has been applied for the proposed planar omnidirectional dipole array design. Figure 2 shows the related simulated and experimental results of the VSWR for the proposed planar dualband dipole array. The related results are listed in Table 1 as a comparison. Results show the satisfactory agreement 

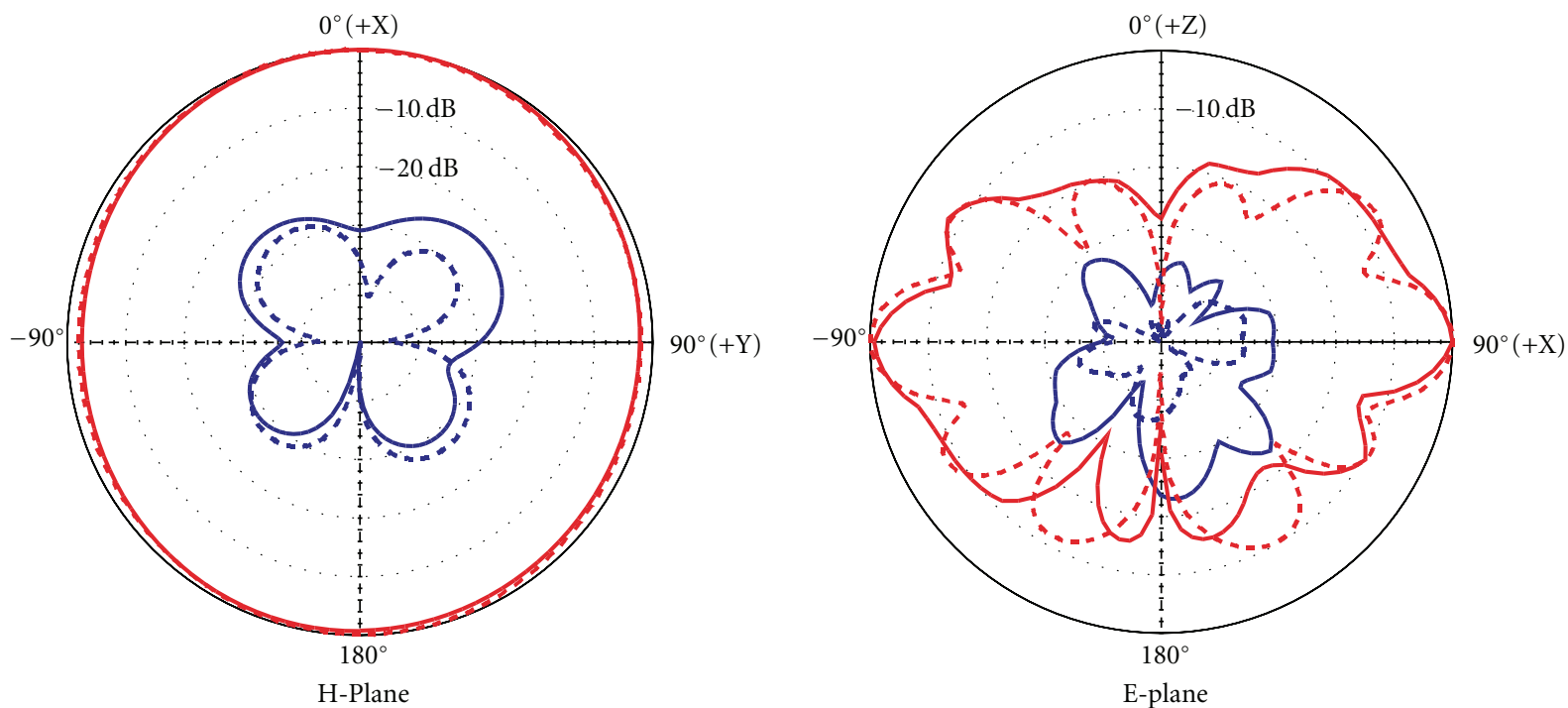

(a)
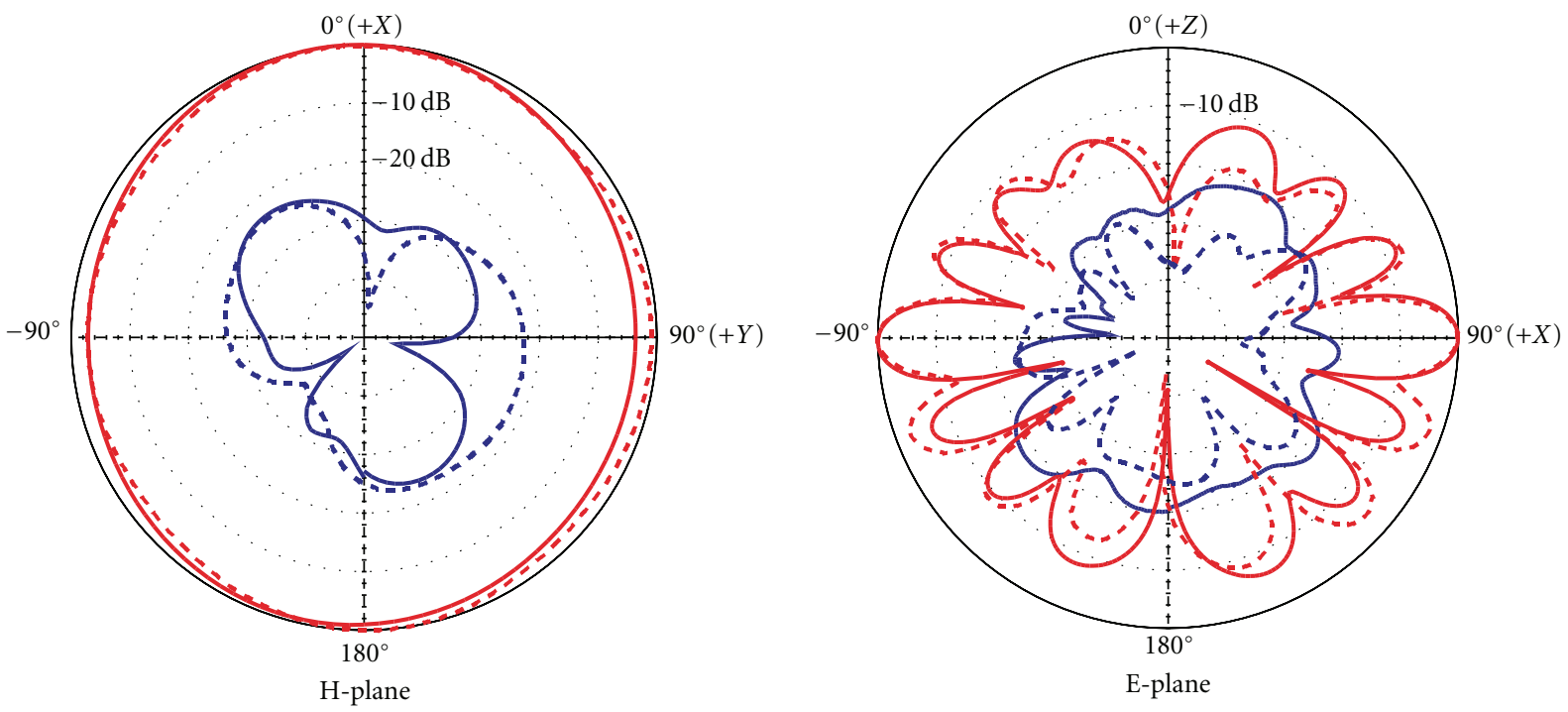

$\begin{array}{ll}-\mathrm{E}_{\theta} & \text { - Measured } \\ -\mathrm{E}_{\phi} & -- \text { Simulated by HFSS }\end{array}$

(b)

FIGURE 4: Simulated and measured 2D radiation patterns for the proposed dual-band dipole array. (a) $f=2600 \mathrm{MHz}$. (b) $f=3500 \mathrm{MHz}$.

for the proposed planar dipole array operating at the 2.6/3.5 GHz bands. From the experimental results, the measured impedance bandwidths (VSWR $\leqq 2.0$ ) can reach $525 / 747 \mathrm{MHz}(20.2 / 21.3 \%)$ for $2.6 / 3.5 \mathrm{GHz}$ bands, respectively, to provide more impedance bandwidth to meet the specifications of LTE and WiMAX systems. The 3D radiation patterns of the proposed dual-band dipole array are measured in anechoic chamber by using NSI-800F with Agilent PNA N5230A. Figure 3 shows the simulated and measured peak gains and efficiencies of the planar dual-band dipole array. The maximum measured peak antenna gains and efficiencies are 7.3/7.5 $\mathrm{dBi}$ and $89 / 75 \%$ across $2.6 / 3.5 \mathrm{GHz}$ bands, respectively. Figure 4 shows the measured 2D radiation patterns of the proposed planar dipole array operating at $2.6 / 3.5 \mathrm{GHz}$ bands. It is found that the radiation patterns are with good omnidirectional radiation patterns in the $\mathrm{X}$ $Y$ plane, which resemble typical patterns in symmetry with respect to the antenna axis $(\theta=0)$ since the proposed dipole array structure is symmetrical.

\section{Conclusions}

A novel planar dual-band dipole array with high-gain operation for LTE/WiMAX access point has been proposed 
and investigated. It provides relatively wider impedance bandwidth of $525 / 747 \mathrm{MHz}(20.2 / 21.3 \%)$ to meet the specifications of LTE $(2.5 \sim 2.7 \mathrm{GHz})$ and WiMAX $(3.3 \sim 3.7 \mathrm{GHz})$ systems, respectively. Also, the proposed dual-band dipole array provides maximum peak antenna gains and efficiencies of $7.3 / 7.5 \mathrm{dBi}$ and $89 / 75 \%$ across the $2.6 / 3.5 \mathrm{GHz}$ operating bands with good omnidirectional radiation pattern in the $\mathrm{X}$ Y plane, respectively.

\section{Acknowledgment}

This paper was supported by the National Science Council (NSC), Taiwan, under Grant NSC97-2221-E-022-005-MY3.

\section{References}

[1] K. L. Wong, F. R. Hsiao, and T. W. Chiou, "Omnidirectional planar dipole array antenna," IEEE Transactions on Antennas and Propagation, vol. 52, no. 2, pp. 624-628, 2004.

[2] Y. T. Liu, T. C. Tseng, and K. L. Wong, "High-gain printed dipole antenna," Microwave and Optical Technology Letters, vol. 46, no. 3, pp. 214-218, 2005.

[3] K. G. Thomas and N. Lenin, "Collinear printed array antenna," Microwave and Optical Technology Letters, vol. 50, no. 6, pp. 1582-1584, 2008.

[4] K. L. Wong, J. W. Lai, and F. R. Hsiao, "Omnidirectional planar dipole-array antenna for $2.4 / 5.2 \mathrm{GHz}$ WLAN access points," Microwave and Optical Technology Letters, vol. 39, no. 1, pp. 3336, 2003.

[5] Ansoft Corporation HFSS, http://www.ansoft.com/products/hf/ hfss/. 

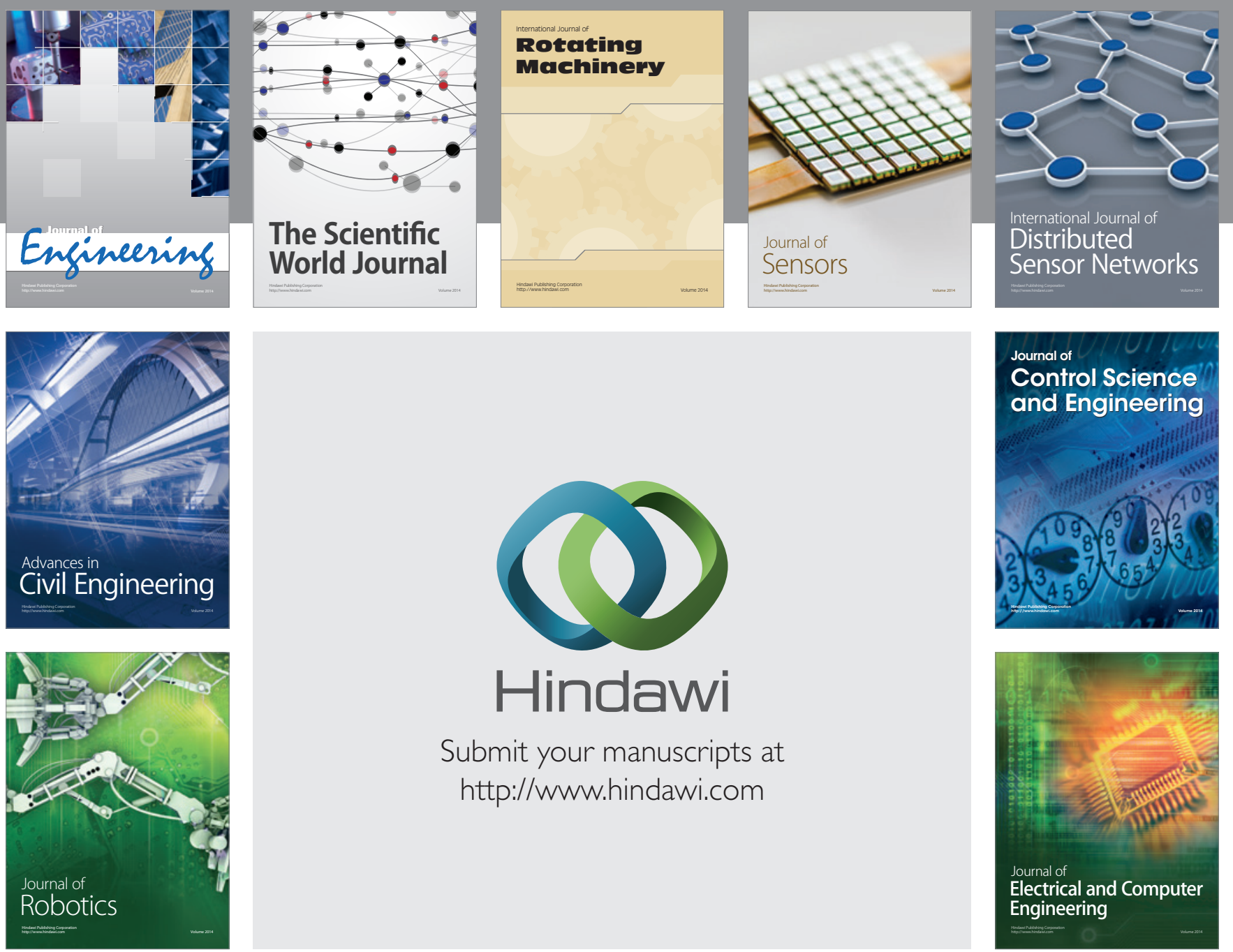

Submit your manuscripts at

http://www.hindawi.com
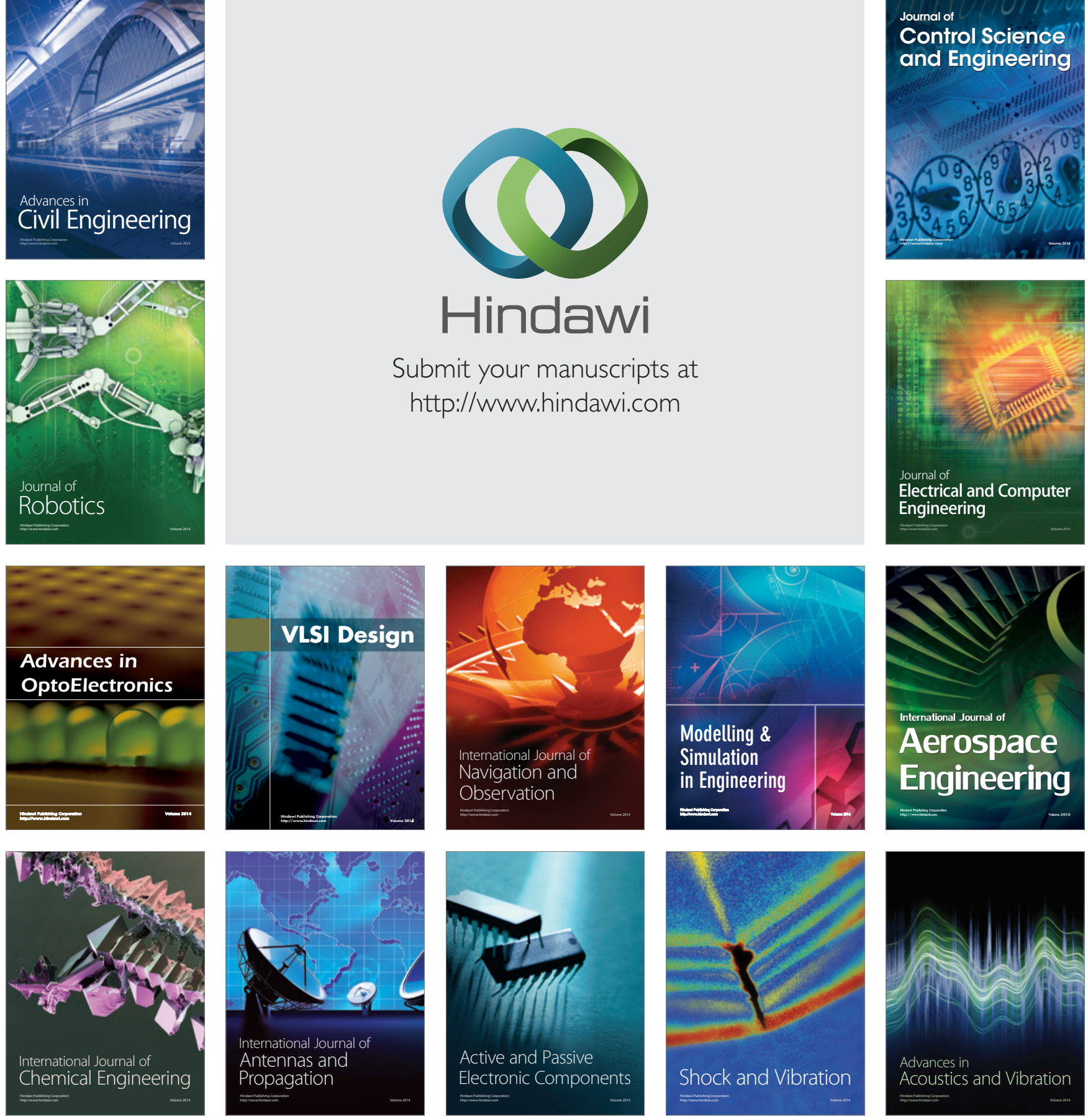